\title{
Transformations de la phénoménologie
}

À propos de Neue Phänomenologie in Frankreich, par Hans-Dieter Gondek et László Tengelyi

\section{Christian Sommer}

\section{(2) OpenEdition}

Journals

Édition électronique

URL : http://journals.openedition.org/rsl/235

DOI : $10.4000 /$ rsl. 235

ISSN : 2271-6246

Éditeur

Éditions Rue d'Ulm

Référence électronique

Christian Sommer, «Transformations de la phénoménologie », Revue Sciences/Lettres [En ligne], 1 ।

2013, mis en ligne le 01 mai 2012, consulté le 22 septembre 2020. URL : http://

journals.openedition.org/rsl/235; DOI : https://doi.org/10.4000/rsl.235

Ce document a été généré automatiquement le 22 septembre 2020.

(c) Revue Sciences/Lettres 


\section{Transformations de la phénoménologie}

À propos de Neue Phänomenologie in Frankreich, par Hans-Dieter Gondek et László Tengelyi

Christian Sommer

\section{RÉFÉRENCE}

Hans-Dieter Gondek et László Tengelyi, Neue Phänomenologie in Frankreich, Berlin, Suhrkamp, 2011.

\section{L'idée de néo-phénoménologie française}

1 S'il est convenu de parler d'une relative éclipse de la phénoménologie dans les années 1960 et 1970, marginalisée par le «structuralisme » et le «néo-structuralisme ${ }^{1}$ », les auteurs du récent et volumineux Neue Phänomenologie in Frankreich, entendent faire l'« inventaire» (Bestandsaufnahme, p. 665), par nature provisoire et lacunaire, de ce qu'ils appellent la "renaissance d'un courant de pensée » (p. 9) depuis le début des années 1980. C'est le premier ouvrage monographique de cette ampleur à risquer une synthèse des évolutions récentes de la phénoménologie en France, dans le sillage de l'ouvrage, qu'il complète et prolonge, de Bernhard Waldenfels, Phänomenologie in Frankreich, publié en $1983^{2}$, sans oublier le modèle commun aux deux, la vaste fresque historique de Herbert Spiegelberg ${ }^{3}$. Gondek et Tengelyi n'ignorent pas les études antérieures sur le sujet qu'ils utilisent au contraire explicitement comme boussoles historiographiques et thématiques pour orienter leur projet, notamment Le Tournant théologique de la phénoménologie française et La Phénoménologie éclatée de Dominique Janicaud ${ }^{4}$, L'Idée de phénoménologie de Jocelyn Benoist ${ }^{5}$, et « Un moment français de la phénoménologie » de Jean-Luc Marion ${ }^{6}$. 
2 Cette «nouvelle phénoménologie » ou néo-phénoménologie pour faire court, est qualifiée de française non seulement parce qu'elle s'énonce dans la langue de Descartes ${ }^{7}$, mais aussi, et surtout, parce qu'elle incarnerait, au-delà d'une définition simplement nationale (p. 32), un moment théorique déterminé de l'histoire de la phénoménologie qui serait un "moment français ». Faisant leur l'aperçu généalogique de Marion esquissé dans "Un moment français de la phénoménologie », Gondek et Tengelyi se laissent guider par « le ou les points communs à la succession ininterrompue qui mène, de Lévinas en 1930, à Ricoeur, Henry et Derrida (et toujours encore Levinas), jusqu'à l'actuelle génération ${ }^{8}$ ». C'est la phénoménologie de cette " génération actuelle » que les auteurs appellent "nouvelle ", car elle soumet à une "transformation essentielle " et "systématique» (p.11, p.666) ses figures précédentes: celle du père fondateur, Husserl, et de son assistant hétérodoxe, Heidegger, mais aussi celle des premiers phénoménologues français, Sartre et Merleau-Ponty. En traçant le diagramme de cette transformation, l'ouvrage apparaît dès lors comme un relais de la complexe et souvent imprévisible dialectique des "transferts et contre-transferts ${ }^{9}$ " dans l'histoire de la philosophie européenne $e^{10}$, ouvrant la possibilité de repérer des problématiques communes, parfois chronologiquement disparates.

3 Comme il ne peut s'agir ici de restituer dans le détail les études et les portraits consacrés aux philosophes jugés représentatifs de la «nouvelle phénoménologie en France $^{11}$ » et destinés à être présentés sous une forme concise et synthétique au public allemand, nous nous concentrons sur la thèse directrice de l'ouvrage : la nouvelle figure de la phénoménologie, dans toutes ses versions, procède d'un changement de paradigme, c'est-à-dire de la modification du concept de phénomène désormais entendu comme événement (Ereignis).

\section{Les trois figures de la phénoménologie}

4 Si le concept de phénomène a subi plus d'une modification en un siècle, Gondek et Tengelyi en proposent une définition minimale censément admise par tous les phénoménologues : le phénomène est une formation de sens (Sinngebilde). Au $§ 55$ des Ideen I, Husserl écrit : "Toutes les unités réelles sont "unités du sens" (Einheiten des Sinnes) », c'est-à-dire qu'elles se donnent comme telles après leur réduction phénoménologique ; il précise aussitôt que ces « unités de sens (...) présupposent une conscience donatrice de sens (sinngebendes Bewusstsein) ${ }^{12}$ ». Les "nouveaux phénoménologues » français, comme avant eux certains élèves de Husserl (Ingarden, Landgrebe, Patocka, Heidegger...), récuseraient ce tournant subjectiviste et idéaliste de Husserl reflété dans cette précision qui lie la réduction phénoménologique et les formations de sens qui en procèdent aux vécus intentionnels ou actes de la conscience.

5 Leur ambition commune, selon Gondek et Tengelyi, est d'étudier des phénomènes ou Sinngebilde qui échappent à la donation de sens déterminée par la conscience intentionnelle et sa structure corrélative noético-noématique (p.23, p.29). De cette ambition commune, marquant un changement de paradigme dans la compréhension du phénomène et, partant, des pratiques de la phénoménologie, on pourrait constater une prise de conscience dans la génération actuelle. Or cette "troisième figure de la phénoménologie » (p.24), qui se détache de la "phénoménologie existentielle» (p. 669) de Sartre (L'Être et le néant, 1943) et du premier Merleau-Ponty (Phénoménologie de la perception, 1945), formée sous la double influence directe de Husserl et Heidegger, 
émerge, en ordre dispersé mais quasi simultané, dès le début des années 1960. Le dernier Merleau-Ponty (Le Visible et l'invisible, 1964; L'œil et l'esprit, 1964), Ricoeur (Philosophie de la volonté II, t. 2, La Symbolique du mal, 1960 ; De l'interprétation. Essai sur Freud, 1965), Henry (L'Essence de la manifestation, 1963) et Lévinas (Totalité et infini, 1961) s'intéressent ainsi, essentiellement, à certains phénomènes contrariant la Sinngebung par la conscience intentionnelle : le «sens spontané » ou "sauvage », l'« invisible », le « symbole », l'« affectivité » ou le « visage ».

6 Pour pratiquer puis exploiter ces percées, la troisième voie de la phénoménologie française passe entre Husserl et Heidegger, voire au-delà (p. 24) : en approchant des phénomènes à contre-courant ou à rebours de la conscience intentionnelle, en donnant voix à des phénomènes de l'«autrement qu'être", la néo-phénoménologie entend explorer des territoires peut-être indiqués par Husserl et Heidegger, mais délaissés et ignorés par eux. Elle ne s'installe pas moins, sur un mode ambivalent, dans la différence entre Husserl et Heidegger, jouant l'un contre l'autre (p. 21, p. 646) : si Heidegger doit servir à critiquer l'ego transcendantal de Husserl et fournir son modèle de lecture ontothéo-logique de l'histoire de la philosophie, le retour à Husserl (p. 645), qui se précisera et consolidera autour de 1981 (Franck, Chair et corps. Sur la phénoménologie de Husserl) et 1984 (Marion, «La percée et l'élargissement. Contribution à l'interprétation des Recherches Logiques de Husserl ", Philosophie, $\mathrm{n}^{\circ} 2$ et 3, repris dans le premier chapitre de Réduction et donation, Paris, PUF, 1989), est sollicité pour résoudre les apories supposées, ou les questions laissées en suspens et contournées, dans l'analytique existentiale de Heidegger (le corps, le sexe, la vie, l'autre, Dieu ...).

7 On peut à cet égard remarquer que les contours de la figure de Heidegger à l'intérieur de la "nouvelle phénoménologie " n'apparaissent pas toujours très clairement dans l'ouvrage. À propos de ce double usage de Husserl et de Heidegger que la «nouvelle phénoménologie " mettrait en œuvre, Gondek et Tengelyi, prolongeant les analyses, citées (p. 641), de Benoist ${ }^{13}$, parlent d'un «post-heideggérianisme» ou «néoheideggérianisme" de la "nouvelle phénoménologie", mêlé d'un husserlianisme hétérodoxe. C'est d'ailleurs moins le Heidegger de Sein und Zeit qui sert de référence, mais singulièrement celui de la conférence de 1962, Temps et être, où s'énoncent, quoique coupés de leur contexte d'élaboration antérieur, quelques concepts majeurs d'après-guerre : le don, l'appel, l'es gibt, le retrait, l'Ereignis, etc. Gondek et Tengelyi n'en restreignent pas moins l'influence de Heidegger dans le champ de la «nouvelle phénoménologie » : par exemple dans le cas de Henry et de Chrétien, mais surtout de Marion, il est vrai que le rapport à Heidegger est indéniablement fort, mais critique ; dans celui de Richir, de Barbaras ou de Depraz et de Benoist, on peut en revanche parler de rupture. Mais parmi les autres protagonistes jugés représentatifs de ce courant figurent Franck, Dastur et Escoubas, dont on ne saurait affirmer qu'ils se sont éloignés de Heidegger; les auteurs estiment pourtant (p.22) que la nouvelle génération, surtout husserlienne, s'est bel et bien réveillée de "l'"hypnose" heideggérienne ${ }^{14} »$.

8 On ne peut que souscrire à ce constat s'il concerne le Heidegger, fragmentaire, fixé par sa réception française dans les premières décennies d'après-guerre, réception parfois sectaire, souvent orientée par l'auto-interprétation rétrospective de Heidegger luimême qui ne rend pas toujours justice au potentiel abrité par son propre travail, surtout celui de la période de Sein und Zeit. Mais il faut sans doute nuancer ce jugement si l'on se souvient que le corpus de Heidegger a considérablement augmenté depuis la 
publication de la Gesamtausgabe et s'est modifié en conséquence, obligeant à réviser tant la compréhension du Heidegger des années vingt, autour de Sein und Zeit de 1927, que celle du Heidegger de l'Ereignis dans l'orbe des Beiträge zur Philosophie de 1936-1938, à ce jour toujours inédits en français ; nous y reviendrons à la fin de ce compte-rendu.

Selon Gondek et Tengelyi, la génération actuelle radicalise et systématise donc les percées de ses aînés au-delà de Husserl et Heidegger - ajoutons plus prudemment : audelà d'un certain Husserl et d'un certain Heidegger - en opérant une transformation du concept même de phénomène (p. 25). Le phénomène excède l'objectivité husserlienne (non-objectif, il déborde l'horizon intentionnel), et l'étantité heideggérienne (nonétant, il se situe dans l'" autrement qu'être »). Cet "excès » propre à la dimension "non-objective» de tout phénomène, aucune ontologie existentiale-analytique ou phénoménologie transcendantale ne sauraient le thématiser (p. 670). Toute approche en revanche qui se donne pour tâche explicite de traiter de cet " excès non-objectif " (ungegenständlicher Überschuss) au cœur du phénomène est qualifiée par les auteurs de "nouvelle phénoménologie » dont les opérations phénoménologiques fondamentales (description, intentionnalité, éidétique, réduction, constitution) se distinguent, par des variations décisives, tant de la figure husserlienne que de la figure sartrienne-merleaupontyenne de la phénoménologie telle qu'elle s'énonce dans l'Avant-Propos de la Phénoménologie de la perception (p. 665-671).

La "nouvelle phénoménologie ", débordant tout horizon intentionnel, voire l'horizon intentionnel de tous les horizons intentionnels, à savoir le monde, n'est plus une "phénoménologie du monde»(Weltphänomenologie, p. 670), mais, selon le terme critique de Janicaud, une "phénoménologie de l'inapparent " (traduction de la notion tardo-heideggérienne das Unscheinbare). Les auteurs réinvestissent la notion de " phénoménologie de l'inapparent » dont ils suspendent la portée initialement critique pour n'en garder que la teneur positive : l'expression ferait signe, désormais, vers une nouvelle normativité du phénomène basée sur la mise en question du concept de phénomène à partir de l'excès inapparent et non-objectif qu'il abriterait toujours déjà (p. 647).

\section{Structure d'une révolution phénoménologique}

Gondek et Tengelyi considèrent que la transformation systématique du concept de phénomène, on l'a dit, définit la «nouveauté » de la troisième figure de la phénoménologie comme "phénoménologie de l'inapparent». Cette transformation, étudiée en détail dans la première partie de l'ouvrage consacrée à Henry, Marion et Richir (p.37-206), implique trois axes thématiques spécifiques, décrits dans la deuxième partie (p. 209-387) également appuyée pour l'essentiel sur Henry, Marion et Richir: le débat autour de la phénoménologie comme une "autre philosophie première ", le projet d'une anthropologie phénoménologique esquissé à partir du rapport entre phénoménologie et psychanalyse, et la controverse sur les limites entre phénoménologie et théologie. La troisième partie (p. 391-485), qui revendique un statut marginal ou digressif dans l'architecture générale de l'ouvrage, traite de Ricoeur et de Derrida sous l'angle de leur contribution à l'émergence de la «nouvelle phénoménologie »; on peut d'ailleurs se demander pourquoi les auteurs n'ont pas jugé utile dans ce contexte de présenter plus longuement Lévinas, de la même génération que Ricoeur, d'autant plus que la publication récente de certains de ses écrits inédits 
invite à une sérieuse correction de perspective de l'œuvre ${ }^{15}$. La dernière partie (p. 487-663) esquisse sept portraits de phénoménologues contemporains (Didier Franck, Françoise Dastur, Éliane Escoubas, Jean-Louis Chrétien, Renaud Barbaras, Natalie Depraz et Jocelyn Benoist) dont les auteurs considèrent qu'ils enrichissent par des éléments nouveaux le concept transformé de phénomène.

pouvoir entrer dans la matière de ces analyses, nous retiendrons surtout que la définition normative nouvelle du phénomène, et là réside l'une des thèses les plus audacieuses des auteurs de l'ouvrage, serait partagée, comme "fondement commun " (p. 39-40), par tous les acteurs du champ de la «nouvelle phénoménologie », aussi et surtout par les deux "pôles extrêmes » (p. 28) figurés par Marion et Richir, selon une opposition déjà mise en avant par Janicaud ${ }^{16}$. En effet l'une des ambitions explicites de Gondek et de Tengelyi, c'est de procéder à un « rééquilibrage » et de présenter en un tableau complet des familles, si l'on peut dire, qui est celui d'« une seule et même époque de la pensée phénoménologique », la «famille phénoménologique » de Marion, avec son contrepoids qui serait incarné par celle de Richir, «nouveau phénoménologue » encore insuffisamment étudié selon les auteurs (p. 19-20). Ces deux «familles " investissent deux positions divergentes face à l'héritage de la génération précédente : une "approche intégrative ", du côté de Marion, qui renoue explicitement avec Lévinas, Ricoeur, Merleau-Ponty, etc., et une « approche différentielle ou polémique » du côté de Richir (p. 25).

13 Le principe même de la phénoménologie s'énonce en fonction de ces positions antipodiques. Marion pose en principe de la phénoménologie: "d'autant plus de réduction, d'autant plus de donation ${ }^{17}$ »; Richir l'exact contraire : «d'autant plus de réduction, d'autant moins de donation ${ }^{18} »$. Pour Marion, mais aussi pour Henry et Chrétien (p. 672), le donné se donne de lui-même à partir de lui-même selon une autodonation qui survient avant toute donation de sens par la conscience intentionnelle. Richir, pour le dire tout aussi schématiquement, récuse ce principe, car la phénoménologie est censée mettre en question ce qui est positivement donné, en montrant comment le donné résulte d'une Sinnstiftung préalable, d'une "institution symbolique » dont il s'agit d'exhiber la genèse ; Janicaud, Barbaras ou encore Benoist attribueraient à la phénoménologie une fonction "critique» similaire (p. 672). Cette genèse du sens ne sollicite pas l'idée d'une constitution transcendantale; le sens est compris comme un événement qui advient spontanément à la conscience intentionnelle.

Dans les deux cas, le phénomène se produit à rebours de toute donation de sens par la conscience intentionnelle, soit qu'il inclut l'«effet de sens» d'une contreintentionnalité (Marion), soit qu'il donne lieu à une formation de sens nonintentionnelle comme "sens se faisant» (Richir). Ainsi les deux principes diamétralement opposés convergent en vérité dans la définition du phénomène comme " événement de sens " (Sinnereignis) qui se donne, "présupposition commune » à ces deux positions extrêmes, et dès lors se rejoignent au-delà du dédoublement empiricotranscendantal (p. 671). C'est donc la thèse du caractère "événementiel »du donné ${ }^{19}$ qui permet aux auteurs d'unifier le champ de la "nouvelle phénoménologie» (p. 672-673). 


\section{Après le tournant théologique} totalité du champ de la néo-phénoménologie française. Les auteurs refusent de parler de «nouveaux théologiens» qui prendraient simplement le masque de la phénoménologie, comme semblait le suggérer Janicaud. Dans leur conclusion, Gondek et Tengelyi n'en insistent pas moins sur la présence continue de thèmes « théologiques » chez les « nouveaux phénoménologues », jusqu'à voir dans l'ensemble de la néo-phénoménologie française opérant après la «mort de Dieu » un réinvestissement philosophique de thèmes initialement théologiques (p. 674), " sécularisation » dont témoigneraient non seulement les notions de don, d'appel ou d'événement chez Henry, Marion et Chrétien, voire chez Derrida, mais aussi les notions de vie, de corps, de chair ou de désir chez Richir et Barbaras. Si la controverse sur les limites entre phénoménologie et théologie a fait l'objet d'un chapitre dans la deuxième partie, cette thèse de la "sécularisation » ou " réactualisation » phénoménologique de thèmes théologiques aurait peut-être gagnée à être davantage explicitée ${ }^{23}$ et inscrite dans un contexte historique plus large.

17 À partir de cette thèse d'une « sécularisation » phénoménologique, Gondek et Tengelyi esquissent dans leur conclusion trois voies possibles pour la néo-phénoménologie et ses protagonistes contemporains. La première voie, empruntée par Marion et Chrétien à la suite de Lévinas, de Henry et de Ricoeur, serait celle d'une « religion post-métaphysique » (p. 674). La deuxième, frayée par le Heidegger tardif et le dernier Merleau-Ponty, pratiquée par Derrida et Richir, mais aussi par Barbaras, conduirait vers une «quasithéologie sans religion » (p. 674). Les auteurs considèrent que Franck, Dastur et Escoubas sont proches de la deuxième voie, Depraz de la première; Benoist chercherait une troisième voie, celle d'un « athéisme non-métaphysique » (p. 675), qui, en interrogeant les limites mêmes de la phénoménalité et de l'intentionnalité (p. 641, p. 658), tracerait une ligne de fuite post-phénoménologique, qui ferait sortir du champ de la «nouvelle phénoménologie ».

18 C'est d'ailleurs un certain hors-champ dans la description de la néo-phénoménologie française que nous voudrions évoquer pour terminer ce bref aperçu. La thèse principale de l'ouvrage, qui sert également de crible pour identifier les «nouveaux phénoménologues ", à savoir le paradigme du phénomène comme "événement de sens ", si elle est susceptible par ailleurs d'être soumise à discussion, présente l'avantage de procurer un principe d'unification du champ de la néo-phénoménologie en France. Mais il reste à montrer que ce paradigme demeure toujours opérationnel si 
on le met à l'épreuve des possibles intersections et interfaces de la néophénoménologie avec d'autres tendances à l'intérieur du champ général de la philosophie française. Soulignons que certaines parmi ces interfaces susceptibles de ressourcer ou d'inscrire la «nouvelle phénoménologie » dans un débat plus vaste, sont au moins indiquées, et les développer n'était évidemment pas du ressort de l'ouvrage déjà volumineux.

En définissant le phénomène, dans son paradigme nouveau, comme "événement de sens ", on aurait pu s'attendre, en effet, que l'ouvrage mette en perspective le rapport complexe entre phénoménologie et herméneutique en France $^{24}$, mais aussi le débat entre phénoménologie et philosophie analytique, frôlé dans le portrait de Benoist sans être approfondi ${ }^{25}$. Autre dossier, l'un des trois axes thématiques principaux procédant du nouveau concept de phénomène serait la constitution d'une anthropologie phénoménologique, mais l'interface entre phénoménologie et « anthropologie » semble uniquement traitée sous l'angle du débat (d'ailleurs bien reconstruit) avec la psychanalyse, alors que cette constellation émerge plus généralement, par exemple dans la réception française en cours, aux effets donc encore imprévisibles, de Blumenberg et de son projet d'anthropologie phénoménologique ${ }^{26}$, lequel, situé au cœur de sa "métaphorologie» comme de sa "mythologie philosophique " et essentiellement lié au corpus de l'anthropologie philosophique allemande (Scheler, Plessner, Gehlen), implique tant une relecture de Husserl que de Heidegger, les deux figures également tutélaires pour la néo-phénoménologie française ${ }^{27}$.

Enfin, dernière remarque, les auteurs soulignent le "rapport spécifique », assurément décisif pour la constitution du champ et de certains de ses thèmes fondamentaux, entre la «nouvelle phénoménologie » et l'histoire de la philosophie (p. 643), à supposer qu'on puisse facilement en faire le partage, et le rôle de Heidegger impliqué par ce rapport. Ce rapport spécifique n'est évoqué que latéralement dans un sous-chapitre sur la réception, par Marion, du théorème heideggérien de la métaphysique comme constitution onto-théo-logique (p. 352-357). Ce théorème, dont on sait qu'il est sans doute tiré de la figure avicenno-scotiste de la métaphysique, a fait récemment l'objet de corrections et de critiques $^{28}$. De là à penser que ces objections, absolument nécessaires, contribuent à désactiver le potentiel de Heidegger pour la phénoménologie contemporaine, il y a un pas qu'il faut se garder de franchir, car c'est sans doute à partir de ces réévaluations, et à la faveur de l'extension considérable du corpus heideggérien au cours du dernier quart de siècle par la Gesamtausgabe, qu'il est possible, par un effet en retour, peut-être moins perceptible car plus micro-logique, de dégager certaines ressources susceptibles d'infléchir ou d'enrichir les problématiques de la "nouvelle phénoménologie " et d'interroger le tracé de ses «familles" françaises ${ }^{29}$ dont l'ouvrage, soulignons-le pour le saluer, propose pour la première fois un tableau d'ensemble qui, loin d'être figé, puisque vivant, ne demande qu'à être complété ou prolongé. 


\section{NOTES}

1. Ce terme importé des États-Unis et diffusé en Allemagne par Manfred Frank (Was ist Neostrukturalismus ?, Francfort, Suhrkamp, 1983, tr. fr. Ch. Berner, Paris, Le Cerf, 1989) et Jürgen Habermas (Der philosophische Diskurs der Moderne, Francfort, Suhrkamp, 1985, tr. fr. Ch. Bouchindhomme et R. Rochlitz, Paris, Gallimard, 2011), est censé désigner principalement les pensées de Foucault, Deleuze, Derrida, Lyotard, ce que d'autres ont pu appeler, avec la même imprécision, la « pensée 68 ».

2. Bernhard Waldenfels, Phänomenologie in Frankreich, Francfort, Suhrkamp, 1983.

3. Herbert Spiegelberg, The Phenomenological Movement: A Historical Introduction (1960), 2 vol. , La Haye, Nijhoff, $3^{\mathrm{e}}$ édition, 1982 ; on se reportera à la $3^{\mathrm{e}}$ partie du $2^{\text {nd }}$ volume, « The French Phase of the Movement », p. 395-592.

4. Le Tournant théologique de la phénoménologie française, Combas, L'Éclat, 1991, et La Phénoménologie éclatée, Combas, L'Éclat, 1998, tous deux repris dans La Phénoménologie dans tous ses états, Paris, Gallimard, 2009.

5. Voir plus particulièrement les trois essais, "Sur l'état présent de la phénoménologie », "Qu'est-ce qui est donné ?", "Le "tournant théologique" ", in L'Idée de phénoménologie, Paris, Beauchesne, 2001, respectivement p. 1-43, p. 45-79, p. 81-103.

6. «Un moment français de la phénoménologie», Rue Descartes, Phénoménologies françaises, 2002/1, n³5, p. 9-13. Les auteurs se réfèrent également à François-David Sebbah, L'Épreuve de la limite. Derrida, Henry, Lévinas et la phénoménologie, Paris, PUF, 2001 ; Michel Haar, La Philosophie française entre phénoménologie et métaphysique, Paris, PUF, 1999 ; B. Bégout, N. Depraz, Ph. Cabestan (éd.), Magazine littéraire, ${ }^{\circ}$ 403, nov. 2001, p. 18-65. François-David Sebbah et Alain David, Rue Descartes, $\mathrm{n}^{\circ}$ 35, 2002/1, p. 1-149; Rudolf Bernet, La Vie du sujet. Recherches sur l'interprétation de Husserl dans la phénoménologie, Paris, PUF, 1994; Conscience et existence. Perspectives phénoménologiques, Paris, PUF, 2004 ; Carla Canullo, La fenomenologia rovesciata. Percorsi tentati in Jean-Luc Marion, Michel Henry e Jean-Louis Chrétien, Turin, Rosenberg \& Sellier, 2004.

7. Vincent Descombes, Le Même et l'autre. Quarante-cinq ans de philosophie française (1933-1978), Paris, Minuit, 1979, p. 11. Sur l'idée de philosophie « française », reconduite à sa (res)source perpétuelle que serait la figure de Descartes, le lecteur pourra se reporter à l'article de Camille Riquier, "Descartes et les trois voies de la philosophie française », Camille Riquier (éd.), Philosophie(s) française(s), Philosophie, $\mathrm{n}^{\circ}$ 109, 2011, p. 21-42.

8. Jean-Luc Marion, art. cité, p. 10.

9. Jean-Claude Monod, "Introduction» au dossier Phénoménologie allemande, phénoménologie française, Revue germanique internationale, 13/2011, Paris, CNRS Éditions, p. 6.

10. La réception germanique de la "nouvelle phénoménologie ", à la différence de sa réception anglo-américaine, est encore balbutiante ; voir par exemple, sur Henry, les travaux de Rolf Kühn, Radikalisierte Phänomenologie, Francfort, P. Lang, 2003 et Michael Staudigl, Die Grenzen der Intentionalität. Zur Kritik der Phänomenalität nach Husserl, Wurtzbourg, K\&N, 2003, ou, sur Marion, l'étude monographique de Thomas Alferi, « Worüber hinaus Grösseres nicht gegeben werden kann... » : Phänomenologie und Offenbarung nach Jean-Luc Marion, Fribourg, Alber, 2007.

11. Jean-Claude Monod et Christian Sommer organisent les mercredi 7 et jeudi 8 mars 2012 deux journées d'étude à propos de la "nouvelle phénoménologie en France " avec la participation de R. Barbaras, J. Benoist, J.-L. Chrétien, J.-F. Courtine, F. Dastur, N. Depraz, É. Escoubas, D. Franck, J.-L. Marion, M. Richir et L. Tengelyi (voir http://www.umr8547.ens.fr/spip.php?rubrique30).

12. Husserl, Ideen zu einer reinen Phänomenologie und phänomenologischen Philosophie, Erstes Buch, Husserliana, III/I, La Hague, Nijhoff, 1976, p. 120.

13. Jocelyn Benoist, «Sur l'état présent de la phénoménologie », L'Idée de phénoménologie, p. 18-20. 
14. La formule est de Marc Richir, Phénoménologie en esquisses. Nouvelles fondations, Grenoble, Millon, 2000, p. 19.

15. Voir Lévinas, Cuvres 1. Carnets de captivité et autres inédits, R. Calin et C. Chalier (éd.), Paris, Grasset/IMEC, 2009 ; CEuvres 2. Parole et silence et autres conférences inédites au Collège philosophique, R. Calin et C. Chalier (éd.), Paris, Grasset/IMEC, 2011.

16. Voir Rue Descartes, Phénoménologies françaises, 2002/1, n 35, p. 145.

17. Jean-Luc Marion, Étant donné. Essai d'une phénoménologie de la donation (1997), Paris, PUF, 2005, p. 7.

18. Marc Richir, "Intentionnalité et intersubjectivité », in D. Janicaud (éd.), L'Intentionnalité en question, Paris, Vrin, 1995, p. 154.

19. Gondeck et Tengelyi renvoient, pour l'élaboration de ce trait caractéristique, à l'essai de J. Benoist, «Qu'est-ce qui est donné ? ", in L'Idée de phénoménologie, p. 45-79.

20. Dans Le Tournant théologique de la phénoménologie française, Combas, L'Éclat, 1991.

21. Jocelyn Benoist, «Sur l'état présent de la phénoménologie », in L'Idée de phénoménologie, p. 24 ; François-David Sebbah, «À l'excès. Un moment de phénoménologie en France », in Jean-Michel Salanskis et François-David Sebbah, Usages contemporains de la phénoménologie, Paris, Sens \& Tonka, 2008, p. 175-208.

22. Par exemple dans son cours de 1928, Gesamtausgabe, tome 26, p. 117, p. 211.

23. Sur l'idée d'une réactualisation phénoménologique de sources patristiques et médiévales, voir Emmanuel Falque, Dieu, la chair et l'autre. D'Irénée à Duns Scot, Paris, PUF, 2008, p. 13-40.

24. Voir Jean Grondin, «La Phénoménologie sans herméneutique », Internationale Zeitschrift für Philosophie, 1, 1992, 146-153 ; Le Tournant herméneutique de la phénoménologie, Paris, PUF, 2003 ; Jean Greisch, Le Cogito herméneutique. L'Herméneutique philosophique et l'héritage cartésien, Paris, Vrin, 2000 ; L'Arbre de vie et l'arbre du savoir. Les racines phénoménologiques de l'herméneutique heideggérienne (1919-1923), Paris, Le Cerf, 2000.

25. Voir sur ce point Jean-Michel Roy, Rhin et Danube. Essais sur le schisme analyticophénoménologique, Paris, Vrin, 2010 ; Claude Romano, Au cœur de la raison, la phénoménologie, Paris, Gallimard, 2010. Remarquons en passant qu'eu égard au critère principal pour définir la néophénoménologie, à savoir la contribution au concept de phénomène comme événement, l'absence, dans l'ouvrage, de l'« herméneutique événementiale " de Claude Romano (déployée dans L'Événement et le monde, Paris, PUF, 1998; L'Événement et le temps, Paris, PUF, 1999 ; Il y a, Paris, PUF, 2003), peut surprendre.

26. Hans Blumenberg, $\mathrm{Zu}$ den Sachen und zurück, Francfort, Suhrkamp, 2002 ; Beschreibung des Menschen, Francfort, Suhrkamp, 2006, Description de l'homme, tr. fr. Denis Trierweiler, Paris, Le Cerf, 2011 ; Jean-Claude Monod, " "L'interdit anthropologique" chez Husserl et Heidegger et sa transgression par Blumenberg", Revue germanique internationale, 2009-10, p. 221-236; Denis Trierweiler (éd.), Hans Blumenberg. Anthropologie philosophique, Paris, PUF, 2010.

27. De cet intérêt anthropologique renouvelé en phénoménologie, qui croise peut-être le "moment du vivant» (Frédéric Worms, La Philosophie en France au XXe siècle. Moments, Paris, Gallimard, 2009, p. 562-563) vers lequel convergent les courants du néo-bergsonisme et du deleuzianisme français axés autour du concept d'événement (M. Rölli (éd.), Ereignis auf Französisch: Von Bergson bis Deleuze, Munich, Fink, 2004), témoigne également l'ouvrage récent d'Étienne Bimbenet, L'animal que je ne suis plus, Paris, Gallimard, 2011, qui, dans un débat avec la philosophie de l'esprit anglo-saxonne, prolonge au-delà de Merleau-Ponty les indications et esquisses d'une anthropologie phénoménologique que celui-ci avait laissées dans La Nature. Notes. Cours du Collège de France, Paris, Le Seuil, 1995. Voir aussi, selon une perspective qui développe certaines pistes élaborées par Richir et Blumenberg, Alexander Schnell, Hinaus. Entwürfe zu einer phänomenologischen Metaphysik und Anthropologie, Wurtzbourg, Königshausen \& Neumann, 2011.

28. Voir Olivier Boulnois, «Heidegger, l’ontothéologie et les structures médiévales de la métaphysique », C. Esposito et P. Porro (éd.), Heidegger e i medievali (Quaestio 1 / 2001), Turnhout, 
Brepols, p. 379-406 ; Jean-François Courtine, "Métaphysique et ontothéologie », in Jean-Marc Narbonne et Luc Langlois (éd.), La Métaphysique. Son histoire, sa critique, ses enjeux, Laval, PUL, 1999, p. 137-157 ; Inventio analogiae, Métaphysique et ontothéologie, Paris, Vrin, 2005 ; Alain de Libera, "Archéologie et reconstruction. Sur la méthode en histoire de la philosophie médiévale », in Karl-Otto Apel et al. (éd.), Un siècle de philosophie 1900-2000, Paris, Gallimard, 2003, p. 583-584.

29. Il faut évoquer sur ce point crucial le travail paradigmatique de Jean-François Courtine, à commencer par Heidegger et la phénoménologie, Paris, Vrin, 1990, jusqu'au récent La Cause de la phénoménologie, Paris, PUF, 2007. Voir aussi Jean-François Courtine (éd.), Heidegger 1919-1929. De l'herméneutique de la facticité à la métaphysique du Dasein, Paris, Vrin, 1996 ; Jean-François Courtine (éd.), L'Introduction à la métaphysique de Heidegger, Paris, Vrin, 2007, et, par ailleurs, les recueils récents procurés par Servanne Jollivet et Claude Romano (éd.), Heidegger en dialogue 1912-1930, Paris, Vrin, 2009, ainsi que par Sophie-Jan Arrien et Sylvain Camilleri (éd.), Le Jeune Heidegger 1909-1926, Paris, Vrin, 2011. 